\title{
Clinical Predictors of Mortality and Critical Illness in Patients with COVID-19 Pneumonia
}

\author{
Maamoun Basheer ${ }^{1, *}$, Elias Saad ${ }^{1,2, *}$, Rechnitzer Hagai ${ }^{3}(\mathbb{D})$ and Nimer Assy ${ }^{1,2, *}$ (D) \\ 1 Internal Medicine Department, Galilee Medical Center, Nahariya 2210001, Israel \\ 2 The Azrieli Faculty of Medicine, Bar-Ilan University, Safad 1311502, Israel \\ 3 The Microbiology Lab, Galilee Medical Center, Nahariya 2210001, Israel; HagaiR@gmc.gov.il \\ * Correspondence: maamon.basheer@mail.huji.ac.il (M.B.); eliass@gmc.gov.il (E.S.); nimera@gmc.gov.il (N.A.)
}

Citation: Basheer, M.; Saad, E.; Hagai, R.; Assy, N. Clinical Predictors of Mortality and Critical Illness in Patients with COVID-19 Pneumonia. Metabolites 2021, 11, 679. https:// doi.org/10.3390/metabo11100679

Academic Editor: Amedeo Lonardo

Received: 20 September 2021 Accepted: 30 September 2021 Published: 2 October 2021

Publisher's Note: MDPI stays neutral with regard to jurisdictional claims in published maps and institutional affiliations.

Copyright: (c) 2021 by the authors. Licensee MDPI, Basel, Switzerland. This article is an open access article distributed under the terms and conditions of the Creative Commons Attribution (CC BY) license (https:// creativecommons.org/licenses/by/ $4.0 /)$.

\begin{abstract}
Early identification of patients with COVID-19 who will develop severe or critical disease symptoms is important for delivering proper and early treatment. We analyzed demographic, clinical, immunological, hematological, biochemical and radiographic findings that may be of utility to clinicians in predicting COVID-19 severity and mortality. Electronic medical record data from patients diagnosed with COVID-19 from November 2020 to June 2021 in the COVID-19 Department in the Galilee Medical Center, Nahariya, Israel, were collected. Epidemiologic, clinical, laboratory and imaging variables were analyzed. Multivariate stepwise regression analyses and discriminant analyses were used to identify and validate powerful predictors. The main outcome measure was invasive ventilation, or death. The study population included 390 patients, with a mean age of $61 \pm 18$, and $51 \%$ were male. The non-survivors were mostly male, elderly and overweight and significantly suffered from hypertension, diabetes mellitus type 2, lung disease, hemodialysis and past use of aspirin. Four predictive factors were found that associated with increased disease severity and/or mortality: age, NLR, BUN, and use of high flow oxygen therapy (HFNC). The AUC or diagnostic accuracy was $87 \%$, with a sensitivity of $97 \%$, specificity of $60 \%$, PPV of $87 \%$ and NPP of $91 \%$. The cytokine levels of CXCL-10, GCSF, IL-2 and IL-6 were significantly reduced upon the discharge of severely ill COVID-19 patients. The predictive factors associated with increased mortality include age, NLR, BUN, and use of HFNC upon admission. Identifying those with higher risks of mortality could help in early interventions to reduce the risk of death.
\end{abstract}

Keywords: SARS-COV-2; NLR; high flow; BUN; insulin resistance; mortality; cytokine storm; predictors

\section{Introduction}

People infected with SARS-CoV-2 may develop COVID-19, which has a wide range of clinical severity, from a mild upper respiratory tract inflammation to a diffuse viral pneumonia causing acute respiratory failure, including lung injury, multiorgan failure, sepsis and death [1-3]. According to the NIH management guidelines, $80 \%$ of the COVID-19 patients worldwide were classified as mild (fever, cough, malaise), 14\% severe (pneumonia and hypoxemia), and 5\% as critical illness such as septic shock and acute respiratory distress syndrome (ARDS) [1-3].

Patients need support for many of the common complications of severe COVID-19, including pneumonia, hypoxemic respiratory failure, ARDS, sepsis, septic shock and acute kidney injury. In addition to the complications directly caused by COVID-19, there are complications from prolonged hospitalizations, which include secondary bacterial infections, thromboembolisms, gastrointestinal bleeding and critical illness [4-8]. Several therapeutic agents have been evaluated for treating COVID-19, such as the antiviral Remdesivir, steroids (e.g., dexamethasone and methyl-prednisone) and anti-IL-1 and anti-IL-6 inhibitors $[9,10]$. The average case fatality rate in China was $2.3 \%$, but mortality was as high as $49 \%$ in patients with critical illness [11]. 
Many studies suggest a template or scores for predicting the prognosis of COVID-19 patients [12-18]. It is difficult to accurately predict clinical outcomes for patients across this spectrum of clinical presentations [12-17], thus making it difficult to manage these patients. An improved understanding of predictive factors for COVID-19 is critical for improving clinical decisions. If physicians can better identify those with higher mortality risks, interventions can be implemented to reduce the risk of death.

We analyzed clinical data of confirmed COVID-19 patients, to see whether we could find predictors of severity and mortality. A group of parameters was found to affect the severity and mortality of the patients. We also identified clinical features that contributed to the disease progression.

\section{Results}

\subsection{Clinical Characteristics of the Surviving and Non-Surviving COVID-19 Patients}

The data of 392 COVID-19 patients were analyzed. Of these patients, 318 were discharged and 74 died. The surviving group was significantly younger than the nonsurvivors (58 \pm 18 vs. $78 \pm 12, p<0.001$, respectively). Of these groups, 60 and $47 \%$ of the non-survivor and the surviving groups were males, respectively. Among the nonsurvivors, the BMI was significantly higher than in those that survived (Table 1). The majority of the non-survivors significantly suffered from hypertension, diabetes mellitus type 2, lung disease, hemodialysis and past medical use of aspirin. The majority of the non-survivors were diagnosed as having severe symptoms upon admission. No differences in the symptoms before admission (diarrhea, fever and dyspnea) were found between the two groups. The differences in the laboratory findings between the two groups included BUN, ALT, absolute lymphocyte count, neutrophil to lymphocyte ratio, triglycerides, inflammation markers (CRP, D-dimer and IL-6) and the percent of patients who suffered from ARDS, cytokine storm and insulin resistance, which was higher in the non-surviving group. Significant differences were seen in the use of $\mathrm{O}_{2}$ upon admission, the use of high flow in the hospitalization periods, mechanical ventilation and the need for $\mathrm{O}_{2}$ supply on day seven, which were higher in the non-surviving group. There were no differences in the COVID-19 treatments during the hospitalization time (Table 1).

Table 1. Clinical characteristics of the surviving and non-surviving patients with COVID-19 infection. NS: not significant $(p$ value $<0.05)$.

\begin{tabular}{|c|c|c|c|}
\hline Variable & Survivors & Non-Survivors & $p$-Value \\
\hline Total & $N=318$ & $N=74$ & \\
\hline Age & $58 \pm 18$ & $78 \pm 12$ & 0.001 \\
\hline Male (\%) & $47 \%$ & $60 \%$ & 600 \\
\hline BMI & $29 \pm 6$ & $32 \pm 11$ & 0.008 \\
\hline \multicolumn{4}{|l|}{ Comorbidities \% } \\
\hline Diabetes (\%) & 30 & 57 & 0.001 \\
\hline Hypertension (\%) & 48 & 84 & 0.001 \\
\hline Lung disease (\%) & 9 & 18 & 0.03 \\
\hline Hemodialysis (\%) & 5 & 15 & 0.003 \\
\hline Aspirin use (\%) & 30 & 61 & 0.001 \\
\hline Symptom's duration before admission to hospitals (days) & $6 \pm 5$ & $9 \pm 12$ & 0.19 \\
\hline \multicolumn{4}{|l|}{$\begin{array}{c}\text { Symptoms before admission } \\
(\% \text { of total })\end{array}$} \\
\hline Fever \% & 57 & 56 & 0.77 \\
\hline Diarrhea \% & 6 & 2 & 0.26 \\
\hline Dyspnea \% & 59 & 59 & 0.74 \\
\hline Clinical severity on admission $\%$ & 30 & 54 & 0.001 \\
\hline
\end{tabular}


Table 1. Cont.

\begin{tabular}{|c|c|c|c|}
\hline Variable & Survivors & Non-Survivors & $p$-Value \\
\hline \multicolumn{4}{|l|}{ Lab Findings upon admission } \\
\hline Hemoglobin (mg/dL) & $13 \pm 3$ & $12 \pm 1.2$ & 0.06 \\
\hline Absolute neutrophil count $\left(\times 10^{3} / \mu \mathrm{L}\right)$ & $13 \pm 9$ & $13 \pm 6$ & 0.9 \\
\hline Absolute lymphocyte count $\left(\times 10^{3} / \mu \mathrm{L}\right)$ & $2.06 \pm 11$ & $1.4 \pm 4$ & 0.001 \\
\hline Neutrophil to lymphocyte ratio (NLR) & $7.01 \pm 3$ & $9.1 \pm 0.8$ & 0.001 \\
\hline Platelet $\left(\times 10^{3} / \mu \mathrm{L}\right)$ & $220 \pm 86$ & $220 \pm 90$ & 0.79 \\
\hline BUN (mg/dL) & $19 \pm 14$ & $40 \pm 27$ & 0.001 \\
\hline Creatinine (mg/dL) & $2 \pm 8$ & $2.2 \pm 2$ & 0.7 \\
\hline Triglycerides (mg/dL) & $145 \pm 148$ & $157 \pm 48$ & 0.08 \\
\hline HDL (mg/dL) & $33 \pm 12$ & $30 \pm 13$ & 0.09 \\
\hline Insulin Resistance (TG/HDL) & $5.4 \pm 5.8$ & $6.1 \pm 5.0$ & 0.02 \\
\hline C-reactive protein (CRP) (mg/dL) & $68 \pm 78$ & $109 \pm 97$ & 0.001 \\
\hline Ferritin & $632 \pm 987$ & $1041 \pm 3594$ & 0.57 \\
\hline D-dimer & $1664 \pm 3513$ & $2539 \pm 3374$ & 0.001 \\
\hline Fibrinogen & $641 \pm 183$ & $660 \pm 180$ & 0.33 \\
\hline IL-6 & $34 \pm 52$ & $264 \pm 111$ & 0.001 \\
\hline ALT & $23 \pm 21$ & $37 \pm 51$ & 0.001 \\
\hline Cytokine storm (\% of total) & 25 & 51 & 0.001 \\
\hline 4-C score & $8 \pm 20$ & $12 \pm 3$ & 0.001 \\
\hline SOFA score & $1.3 \pm 1.4$ & $2.7 \pm 2.2$ & 0.001 \\
\hline $\mathrm{O}_{2}$ supplement on admission $\%$ & 55 & 100 & 0.001 \\
\hline High flow use (\% of total) & 14 & 82 & 0.001 \\
\hline Mechanical ventilation ( $\%$ of total) & 2 & 46 & 0.001 \\
\hline ARDS (\% of total) & 17 & 34 & 0.006 \\
\hline $\mathrm{O}_{2}$ supply at day 7 (\% of total) & 18 & 99 & 0.001 \\
\hline Hospitalization length & $9.5 \pm 10$ & $17.2 \pm 13$ & 0.001 \\
\hline Time to negative PCR & $17 \pm 8$ & $20 \pm 8$ & 0.16 \\
\hline \multicolumn{4}{|l|}{ Treatment in hospitalization } \\
\hline Steroid therapy (\% of total) & 93 & 97 & 0.26 \\
\hline Methylprednisolone & 86 & 90 & 0.75 \\
\hline Dexamethasone & 14 & 10 & 0.75 \\
\hline LMWH (\% of total) & 96 & 99 & 0.34 \\
\hline Remdesivir (\% of total) & 12 & 19 & 0.21 \\
\hline Vitamin D (\% of total) & 97 & 97 & 0.9 \\
\hline
\end{tabular}

\subsection{Association between Risk Factors and Mortality}

Risk factors such as age, gender, ethnicity, BMI and past medical history, including diabetes mellitus type 2, hypertension, lung disease, chronic kidney disease and hemodialysis, were studied for their correlation to mortality in the COVID-19 patients. The results show that being elderly and hemodialysis were the major risk factors for mortality in the COVID-19 patients (Table 2A). A multi-regression analysis of risk factors showed that age is the main risk factor. However, hemodialysis and a high BMI were also major risk factors for mortality in the COVID-19 patients (Table 2B). 
Table 2. Correlations between risk factors and mortality in COVID-19 patients. (A): Univariate analysis of the strength of risk factors in mortality prediction. (B): Multiregression analysis of risk factors. (C): Analysis of past medical history of the patients and mortality risks.

\begin{tabular}{|c|c|c|c|c|c|}
\hline A & & & & & \\
\hline & Coefficient & 95\%Conf. $( \pm)$ & Std. Error & $\mathrm{T}$ & $p$-Value \\
\hline \multicolumn{6}{|l|}{ Constant } \\
\hline Age & 0.01 & 0.002 & 0.001 & 5.4 & 0.00001 \\
\hline Gender & -0.04 & 0.07 & 0.036 & -1.36 & 0.17 \\
\hline Ethnicity & -0.04 & 0.07 & 0.03 & -1.23 & 0.21 \\
\hline BMI & 0.001 & 0.005 & 0.002 & 2.23 & 0.02 \\
\hline DM & 0.04 & 0.088 & 0.044 & 1.05 & 0.2 \\
\hline HTN & -0.02 & 0.048 & 0.048 & 0.5 & 0.6 \\
\hline Hemodialysis & 0.1 & 0.07 & 0.07 & 2.2 & 0.02 \\
\hline \multicolumn{6}{|l|}{ B } \\
\hline & Coefficient & 95\%Conf. ( \pm ) & Std. Error & $\mathrm{T}$ & $p$-Value \\
\hline \multicolumn{6}{|l|}{ Constant } \\
\hline Age & 0.01 & 0.001 & 0.001 & 7.7 & 0.00001 \\
\hline BMI & 0.01 & 0.005 & 0.0025 & 2.3 & 0.00001 \\
\hline Hemodialysis & 0.176 & 0.13 & 0.069 & 2.5 & 0.00001 \\
\hline \multicolumn{6}{|l|}{ C } \\
\hline & Coefficient & 95\%Conf. $( \pm)$ & Std. Error & $\mathbf{T}$ & $p$-Value \\
\hline \multicolumn{6}{|l|}{ Constant } \\
\hline Past Aspirin use & 0.14 & 0.08 & 0.04 & 3.6 & 0.0003 \\
\hline $\mathrm{O}_{2}$ supplement before admission & 0.25 & 0.08 & 0.04 & 6.2 & 0.00001 \\
\hline
\end{tabular}

Abbreviations: BMI body mass index; DM, type 2 diabetes mellitus; HTN, hypertension. Abbreviation: SE are the standard errors of the regression coefficients. T is the quotient of the coefficient. Two-sided $p$ values or observed significance levels.

Analysis of past medical history treatments showed that aspirin use is a major risk factor for COVID-19 mortality. Furthermore, the patients who received home oxygen supply before admission had a higher risk of mortality (Table 2C). No difference was found in the pre-admission symptoms such as fever, dyspnea and diarrhea, weakness, myalgia and saturation at admission.

\subsection{Correlation between Blood Tests, Severity of Pneumonia and Mortality in COVID-19 Patients}

The clinical parameters and blood tests upon admission of the COVID-19 patients included $\mathrm{PAO} 2 / \mathrm{FiO} 2$, extent of lung injury (pneumonia), fibrinogen, D-dimer, platelet count, mean platelet volume (MPV), neutrophil to lymphocyte ratio (NLR), ferritin, ALT, AST, BUN, creatinine, sodium, phosphor, HDL and triglycerides. These parameters were tested for their correlation to mortality. Univariate analyses show a correlation between age, ARDS and NLR (Table 3A). Multi-regression analyses show significant correlations between age, NLR, BUN and high flow use upon admission and mortality in COVID-19 patients. The non-survivors were elderly, had high BUN, NLR and used high flow ventilation upon admission. These parameters were significantly associated with mortality in the severe COVID-19 patients (Table 3B). 
Table 3. (A): Univariate analyses of the strength of clinical and laboratory tests in predicting mortality in COVID-19 patients. (B): Multiregression analyses of the clinical parameters, blood tests and their correlation to mortality in COVID-19 patients.

\begin{tabular}{|c|c|c|c|c|c|}
\hline A & & & & & \\
\hline & Coefficient & 95\%Conf. $( \pm)$ & Std. Error & $\mathrm{T}$ & $p$-Value \\
\hline \multicolumn{6}{|l|}{ Constant } \\
\hline Age & 0.01 & 0.01 & 0.008 & 1.35 & 0.18 \\
\hline BMI & 0.008 & 0.024 & 0.01 & 0.89 & 0.38 \\
\hline $\mathrm{SO}_{2}$ upon admission & -0.004 & 0.044 & 0.02 & -0.26 & 0.79 \\
\hline$\%$ Pneumonia & 0.0003 & 0.007 & 0.003 & 0.104 & 0.9 \\
\hline $\mathrm{PAO}_{2} / \mathrm{FIO}_{2}$ & 0.0002 & 0.001 & 0.0004 & 0.42 & 0.67 \\
\hline ARDS class & 0.187 & 0.37 & 0.18 & 1.043 & 0.3 \\
\hline D-Dimer & -0.0001 & 8.6 & 0.001 & -0.50 & 0.62 \\
\hline Fibrinogen & -0.000 & 0.0007 & 0.001 & -1.26 & 0.21 \\
\hline NLR & 0.00125 & 0.0014 & 0.001 & 1.84 & 0.076 \\
\hline Ferritin & 0.001 & 0.0001 & 0.001 & 0.75 & 0.45 \\
\hline IL-6 & 0.0007 & 0.002 & 0.001 & 0.54 & 0.58 \\
\hline ALT & -0.001 & 0.007 & 0.001 & -0.51 & 0.61 \\
\hline BUN & 0.004 & 0.014 & 0.007 & 0.56 & 0.56 \\
\hline BUN Class & 0.096 & 0.36 & 0.175 & 0.557 & 0.58 \\
\hline Cytokine storme & -0.068 & 0.33 & 0.16 & -0.427 & 0.67 \\
\hline CRP & 0.0001 & 0.002 & 0.001 & 0.1 & 0.92 \\
\hline HDL & -0.006 & 0.016 & 0.008 & -0.81 & 0.42 \\
\hline HDL Class & 0.003 & 0.34 & 0.169 & 0.01 & 0.98 \\
\hline TG & -0.0004 & 0.002 & 0.0014 & -0.29 & 0.76 \\
\hline TG/HDL & -0.0004 & 0.055 & 0.026 & -0.017 & 0.98 \\
\hline 4C-score & -0.012 & 0.07 & 0.03 & -0.36 & 0.71 \\
\hline High flow use & 0.18 & 0.38 & 0.18 & 0.96 & 0.34 \\
\hline \multicolumn{6}{|l|}{ B } \\
\hline & Coefficient & 95\%Conf. ( $( \pm)$ & Std. Error & $\mathbf{T}$ & $p$-Value \\
\hline \multicolumn{6}{|l|}{ Constant } \\
\hline Age & 0.009 & 0.005 & 0.002 & 3.1 & 0.003 \\
\hline NLR & 0.001 & 0.001 & 0.0005 & 2.3 & 0.022 \\
\hline BUN & 0.006 & 0.004 & 0.002 & 2.9 & 0.004 \\
\hline High flow use & 0.258 & 0.183 & 0.091 & 2.8 & 0.006 \\
\hline
\end{tabular}

Abbreviation: SE are the standard errors of the regression coefficients. T is the quotient of the coefficient. Two-sided $p$ values or observed significance levels.

2.4. Discriminant Analysis with Diagnostic Accuracy of the Correlations between Clinical Parameters and Survival

There is a good correlation between age, NLR, BUN and the use of high flow upon admission, and disease severity and mortality in the COVID-19 patients. The sensitivity, specificity, positive predictive value and negative predictive value are 97.9, 59.7, 86.7 and $91.4 \%$, respectively (Table $4 \mathrm{~A}, \mathrm{~B})$. 
Table 4. The validity (predictive power) of age, NLR, BUN, and use of HFNC, and mortality in COVID-19 patients. The accuracy of the calculations is $87 \%$. (A): The number of samples: Predicted condition-218, survival; 47, death; and true condition, 0 for disease and 1 for no disease. (B): the sensitivity, specificity, positive predictive value and negative predictive value.

\begin{tabular}{cccc}
\hline A & & & \\
\hline & Actual count & 0 & 1 \\
\hline 218 & 189 & 29 \\
\hline 47 & 4 & 43 \\
\hline B & & 59.7 \\
\hline & Specificity & 97.9 \\
\hline & Sensitivity & 86.7 \\
\hline & Positive predictive value & 91.5 \\
\hline & Negative predictive value &
\end{tabular}

2.5. Correlation between the 4-C Score, Cytokines Storm Syndrome, Liver Injury, ARDS and Mortality in COVID-19 Patients

Correlations between BUN classes, cytokine storm syndrome, PAo2/Fio2, 4-C score, liver injury and severity and mortality of the disease is shown in Figure 1. A high 4-C score shows good correlation with the cytokine storm syndrome and ARDS (Figure 1A) with the mortality being higher in this group. A high 4-C score and a decrease in the PAO2/FiO2 ratio correlates with high mortality (Figure 1B). Liver injury and high BUN upon admission correlates with the 4-C score and mortality, as seen in Figure $1 C, D$, respectively.
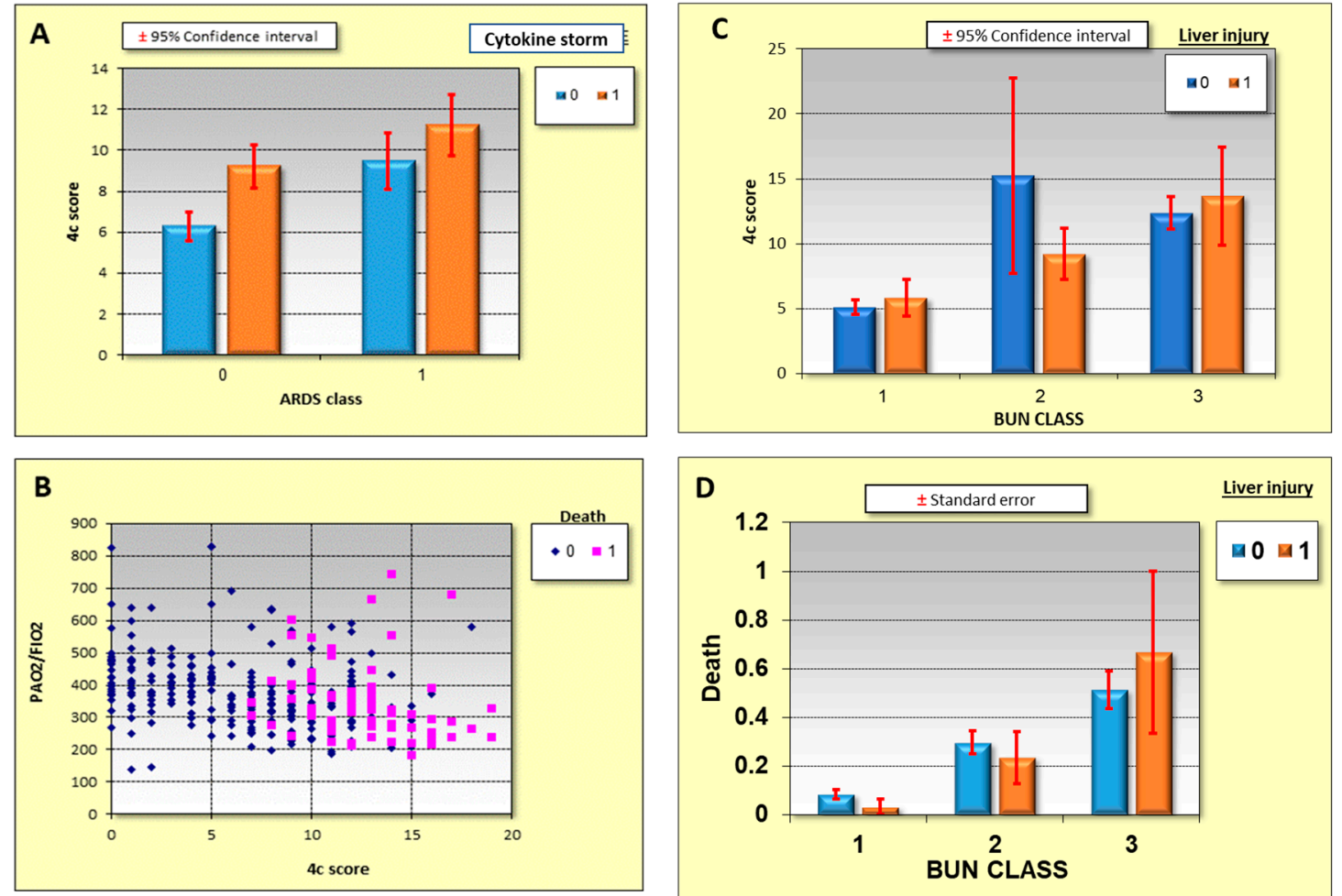

Figure 1. Correlations between ARDS, liver injury, PAO2/FiO2, the 4-C score, and mortality in COVID-19 patients. (A): Correlation between the 4-C score, cytokine storm syndrome and ARDS. (B): Correlation between the 4-C score, liver injury and BUN classes. (C): Correlation between the 4-C score and a decreased $\mathrm{PAO} 2 / \mathrm{FiO} 2$ ratio and high mortality. (D): Correlation between liver injury, BUN class and high mortality. Standart deviation added to (A,C,D). 


\subsection{Kaplan Meir Survival Analysis According to HDL Classes}

Kaplan-Meier analysis showed that the length of hospitalization is correlated with mortality in the COVID-19 patients. Long periods of hospitalization are a major risk of mortality (Figure 2). Survival analysis versus hospitalization length using the Cox regression confirms this relationship.

\section{Survival analysis according to Cox regression}

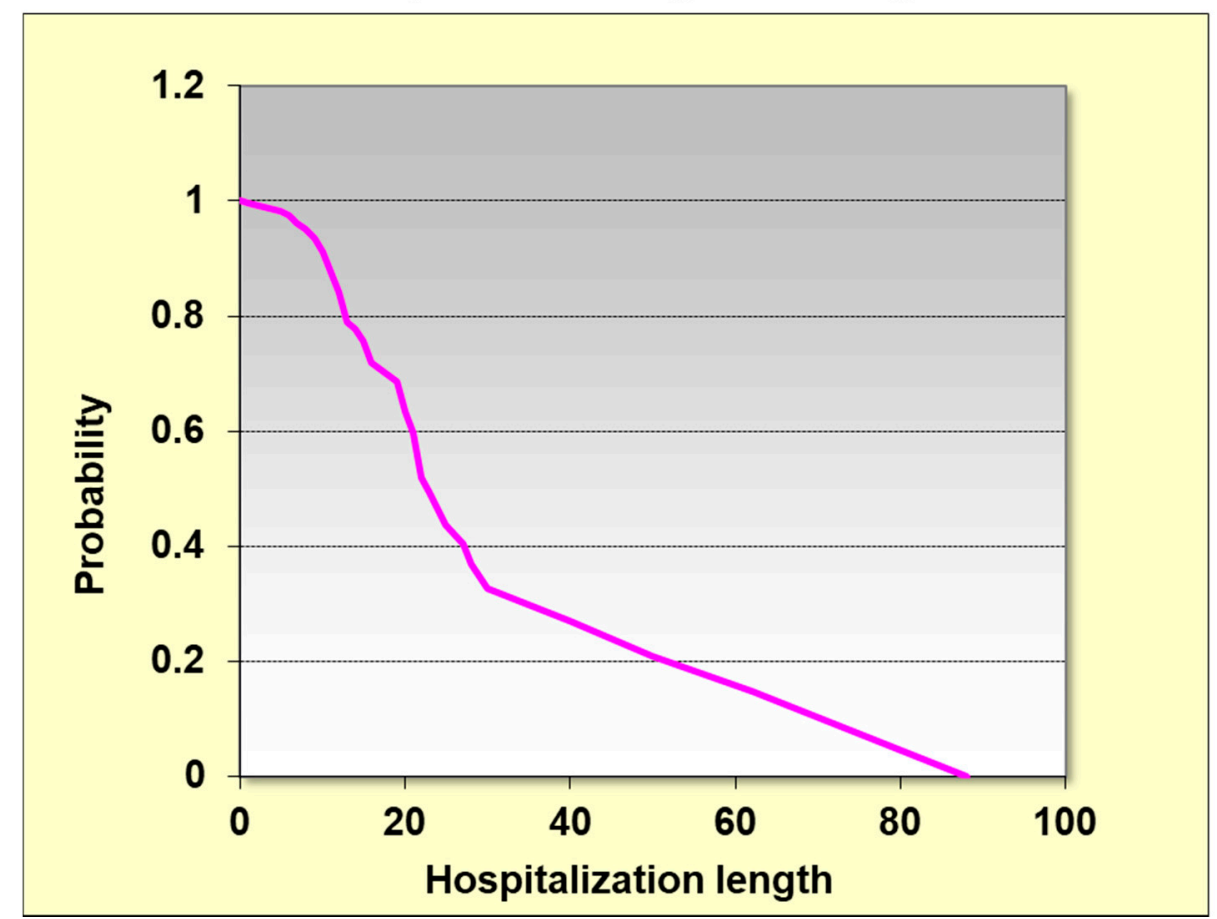

Figure 2. Survival analysis versus hospitalization length using Cox regression.

2.7. Pro-Inflammatory Cytokine Concentrations in COVID-19 Patients: Upon Admission vs. upon Discharge, Severe vs. Mild COVID-19 Patients, Survivors vs. Non-Survivors

Serum pro-inflammatory cytokine levels were measured upon admission and again upon discharge. CXCL-10, GCSF, IL-2 and IL-6 serum concentrations were significantly reduced upon discharge, as seen in Figure 3A. A comparison of these cytokines between the severely ill patients who died during their hospitalization period and the mildly ill patients who were discharged two days later was conducted. The concentrations of CXCL-10, IL-2 and IL-6 were significantly elevated in the non-surviving group (Figure 3B). 


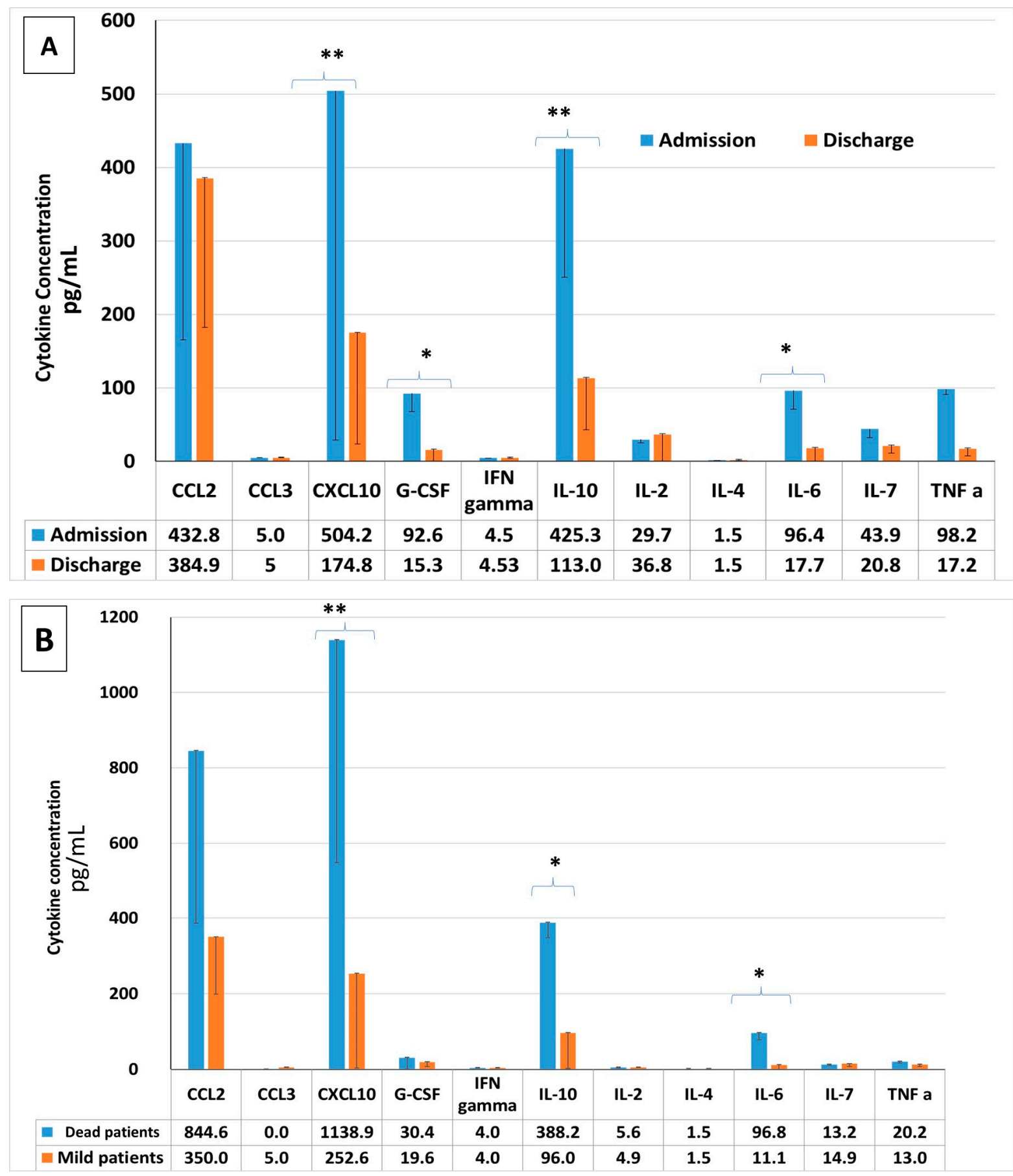

Figure 3. Cytokines levels in COVID-19 patients: The cytokines measured are CCL-2, CCL-3, CXCL-10, GCSF, IFN-gamma, IL-10, IL-2, IL-4, IL-6, IL-7 and TNF $\alpha$. Cytokine levels were measured upon admission and upon discharge, in severely ill and mildly ill patients, and also between survivors and those not surviving. (A): Cytokine levels upon admission vs. upon discharge. (B) survivors vs. non-survivors. ${ }^{*} p<0.05$, ${ }^{* *} p<0.001$.

\section{Discussion}

Understanding the predictive factors for COVID-19 is critical for improving clinical decisions. Our study clearly indicates that age, NLR, BUN and use of high flow oxygen therapy upon admission are predictive factors associated with increased mortality in these patients. Identifying those with higher mortality risks can allow physicians to make early interventions and reduce the risk of death. 
We found that age correlates with the severity of COVID-19 disease. Elderly patients are associated with elevated levels of hypertension and diabetes mellitus. This is due to stiffer vessels and impaired metabolic efficiency. This could explain the mechanism underlying the risk of being morbidly obese and elderly in COVID-19 [19-22]. These data are comparable with the data from the CDC about the severity of the disease in the elderly $[19,20]$.

Our study also shows that high NLR correlates with the disease severity and mortality of the COVID-19 patients. The human immune response, induced by a viral infection, is primarily associated with lymphocytes. Systematic inflammation significantly reduces CD4+ T lymphocytes, and increases suppressive CD8+ T lymphocytes, thus significantly reducing cellular immunity [23]. For this reason, the inflammation caused by the virus increases the NLR [23]. A SARS-COV-2 virus infection may cause a massive reduction in the serum lymphocyte concentration, and therefore, a high NLR could be a predictor of disease severity.

High levels of blood urea nitrogen (BUN) upon admission showed a direct correlation with mortality in our COVID-19 patients. BUN is the end product of nitrogen metabolism and is partially reabsorbed from renal tubules [24-27]. Coronavirus can affect kidney function by entering kidney cells in a direct ACE2-dependent way and activate the reninangiotensin-aldosterone system $[26,27]$. These systemic effects cause renal vasoconstriction, and consequently, glomerular filtration, causing a reduction in BUN excretion [26,27]. Additionally, high levels of inflammation in severe COVID-19 patients perturbs the reninangiotensin system [27]. Therefore, BUN is a good marker of a high inflammation status and may be a good predictor of the severity of the disease.

A direct correlation was found between the use of high flow upon admission and a high mortality rate. The use of high flow means that the patient has a decreased lung capacity and an increased percent of lung injury with hypoxemia. It is also known that hypoxia, along with other high comorbidities, cause poor outcomes [28]. In the initial period of the disease, hypoxic respiratory failure was evaluated as typical ARDS [28]. However, other findings suggest that moderate-to-severely hypoxemic patients affected by COVID-19 may benefit from high flow use and could potentially decrease the need and duration of mechanical ventilation and ICU length of stay without a negative impact in hospital mortality [29]. This possibly may depend on the patients themselves and the intensity of the lung injury. For patients with broad pulmonary involvement and risk factors, as described above, HF is less likely to prevent ventilation, while those with low pulmonary involvement and without risk factors are more likely to improve.

The surviving group in our study were younger than the non-survivors and the majority of the non-survivors were overweight and male. The majority of the non-survivors significantly suffered from hypertension, diabetes mellitus type 2, lung disease, hemodialysis and were diagnosed as severe patients upon admission. Obesity is causally related to metabolic syndrome, which presents as hypertension, diabetes mellitus, coronary heart disease, stroke, renal disease and heart failure [21]. Obesity or excess ectopic fat deposition may be a unifying risk factor for severe COVID-19 infection, reducing protective cardiorespiratory reserve as well as causing immune dysregulation $[20,21]$.

Inflammation markers (CRP, D-dimer and IL-6) were significantly higher in the nonsurvivor groups. The cytokine levels of CXCL-10, GCSF, IL-2 and IL-6 were also significantly higher upon admission of the severely ill patients and in the critically ill patients as well. The cytokine storm is the result of the immune response to the virus and it is a possible major mortality risk factor in COVID-19 patients. The mildly ill patients had low concentrations of these pro-inflammation cytokines. Accumulating evidence suggests that a subgroup of patients with severe COVID-19 develop this cytokine storm with high levels of pro-inflammatory cytokines and chemokines that are associated with pulmonary inflammation and extensive lung damage [30]. This increases the severity and the mortality of the disease [31].

The majority of the non-survivors in our study used aspirin before admission. The benefits of aspirin in critically ill COVID-19 patients are controversial. One study suggests 
that systemic anticoagulant usage reduces mortality in mechanically ventilated COVID-19 patients [32]. In ARDS, aspirin has been studied with mixed results, where some studies have demonstrated benefit and others have not [33-38]. Patients who have been using aspirin prior to being infected are patients who likely suffer from cardiovascular diseases, and therefore, a COVID-19 infection could be fatal due to the underlying cardiovascular disease and not the aspirin.

This study confirmed that the 4-C score is a good predictor for the severity of the disease. The 4-C score correlates well with the elevation in BUN, ARDS progression and liver injury. Elevated 4-C scores were correlated with mortality in the COVID-19 patients. The 4-C mortality score uses patient demographics, clinical observations, and blood parameters that are commonly available at the time of hospital admission and can accurately characterize the population of hospital patients at high risk of death [19].

Our study has some limitations. The data presented here were largely obtained from reports that emerged early during the COVID-19 pandemic. In addition, the wide diversity of study methodologies, statistical approaches, modest sample sizes and geographic sites may have confounded our interpretation of the data. Nevertheless, in the context of a severe pandemic caused by a novel virus, it is vital to address knowledge gaps in the field and identify factors that are potentially predictive of COVID-19 complications that warrant further investigation. Additional big studies of the COVID-19 risk predictors outside Israel should be completed.

\section{Methods}

\subsection{Study Population}

Electronic medical record (EMR) data from patients diagnosed with COVID-19 from November 2020 to June 2021, in the Galilee Medical Center COVID-19 Department, Nahariya, Israel, were used as the database. COVID-19 diagnosis was based on positive polymerase chain reaction (PCR)-based clinical laboratory testing for the SARS-CoV-2 virus. EMR data from 392 patients were analyzed. Of them, 318 were discharged and 74 died.

\subsection{Study Design}

A retrospective analysis of the EMR data from the COVID-19 patients was performed. The analyzed parameters were demographic background, past medical history and treatments, weight, BMI, symptoms before admission (fever, myalgia, dyspnea and diarrhea), blood laboratory tests (biochemistry, $\mathrm{CBC}$, blood gases, blood type, coagulation tests and inflammatory markers), time to discharge or time to death, time to negative nasopharynges RT-PCR test and treatments upon hospitalization. A COVID-19 diagnosis was confirmed using positive real-time PCR assays from nasal and nasopharyngeal swab specimens. Cytokine storms were defined as described by Caricchio et al. [19]. Blood urea nitrogen (BUN) was classified into 3 groups, $\leq 20,20-40$ and $\geq 40$ (mg/dL). The $4-C$ score was calculated as described by Knight et al. [18]. This score contains parameters such as age, sex at birth, number of comorbidities, respiratory rate on admission, peripheral saturation in room air, Glasgow coma scale, urea and CRP. The sequential organ failure assessment (SOFA) score was measured as individual scores for each organ, to determine progression of organ dysfunction.

\subsection{Luminex-Based Multiplex Assay for Serum Cytokine Concentration}

Serum cytokine concentrations were measured including CCL-2, CCL-3, CXCL-10, GCSF, IFN-gamma, IL-10, IL-2, IL-4, IL-6, IL-7 and TNF $\alpha$. Blood samples of patients were collected upon admission and discharge. Altogether, an analysis of 15 samples was conducted. Samples from five severely ill patients upon admission and five samples of patients who died were compared to five samples of mildly ill patients.

Blood samples were withdrawn from mildly ill and severe patients upon admission and discharge for the mildly ill patients and incubated for $30 \mathrm{~min}$ at room temperature. After coagulation, the blood samples were centrifuged at $1500 \times \mathrm{g}$ at $4{ }^{\circ} \mathrm{C}$ for $15 \mathrm{~min}$ and the serum was separated and aliquoted into 2-milliliter tubes and stored in a $-80^{\circ} \mathrm{C}$ freezer. 
For the cytokine testing, the serum was thawed on ice and serum was pipetted in cryotubes until the assay was performed. To assess serum cytokine levels, the human high sensitivity cytokine Luminex custom 8-plex kits (R\&D Systems, Inc., Minneapolis, MN, USA) were used. Testing was performed in 96-well plates according to the manufacturer's instructions. Test samples were run in singles, while standard samples were run in duplicates. In brief, color-coded superparamagnetic beads coated with analyte-specific antibodies were utilized using the Luminex assay. Beads that recognize different target analytes were mixed together and incubated with the serum sample. Captured analytes were subsequently detected using a cocktail of biotinylated detection antibodies conjugated to streptavidinphycoerythrin. The magnetic beads were then isolated and measured using the Luminex MAGPIX ${ }^{\circledR}$ Analyzer (R\&D Systems, Inc., Minneapolis, MN, USA).

\subsection{Ethics}

This study was approved by our medical center's local ethics committee (N 231-20). Retrospective analysis of data from our electronic medical record database was performed under the oversight of the ICH guidelines for good clinical practice.

\subsection{Outcomes}

We defined severity on admission according to the 4-C score. A 4-C score above 10 was considered severe and accounted for a 30\% probability of death. We defined critical COVID-19 illness as a composite of admission to the intensive care unit, invasive mechanical ventilation, ARDS or death.

\subsection{Statistical Analysis}

Statistical analysis was performed using the WinSTAT program. Results are presented as mean + SD for continuous variables. For categorical variables, the frequency and corresponding diagnosis percentage are provided. The Spearman test was used for correlations between two quantitative variables. Univariate direct regression analysis and multivariate stepwise regression analysis were performed for individual variables, including clinical and biochemical variables as an independent variable, and survival or death as the dependent variable. The diagnostic validity of the powerful predictors (age, BUN, neutrophil to lymphocyte ratio (NLR), 4-C score, high-flow) was tested regarding correct classification of death using discriminant analysis. The percentage of cases correctly classified by each diagnostic test as well as the performance measures of sensitivity, specificity, and positive and negative predictive values were calculated using discriminant analysis. Survival analysis versus hospitalization length according to Cox regression, and Kaplan-Meier survival analysis according to HDL classes, were recorded. Tests of significance were two-tailed, with a significance level of less than 0.05 . WinSTAT is the statistical add-on program for Microsoft Excel (Kalmia Co., California, MA, USA).

\section{Conclusions}

Many clinical characteristics are associated with increased disease severity and/or mortality, including age, NLR, BUN, and use of high flow oxygen therapy. Identifying those with a higher risk of mortality could help in making the right interventions during admission and reduce unwanted outcomes.

Author Contributions: Conceptualization, M.B., E.S., R.H. and N.A.; methodology, M.B.; software, M.B. and N.A.; validation, M.B. and N.A.; formal analysis, M.B. and N.A.; investigation M.B., E.S., R.H. and N.A.; resources, M.B. and N.A.; data curation, M.B. and N.A.; writing-original draft preparation, M.B. and N.A.; writing-review and editing, M.B. and N.A.; visualization, M.B. and N.A.; supervision, N.A.; project administration, M.B. and N.A.; funding acquisition, N.A.; All authors have read and agreed to the published version of the manuscript.

Funding: This study did not receive any specific grants from funding agencies in the public, commercial or not-for-profit sectors. 
Institutional Review Board Statement: The study was conducted according to the guidelines of the Declaration of Helsinki, and approved by medical center's local ethics committee (N 231-20).

Informed Consent Statement: Informed consent was obtained from all subjects involved in the study.

Data Availability Statement: The data presented in this study are available on request from the corresponding author. The data are not publicly available due to privacy of the patient.

Acknowledgments: We would to thank the directors and medical teams of the Internal Medicine Departments in Galilee Medical Center, Nahariya, Israel for their help and support.

Conflicts of Interest: The authors declare no conflict of interest.

\section{Abbreviations}

BUN: Blood urea nitrogen; NLR: Neutrophil to lymphocyte ratio; HFNC: High flow nasal cannula.

\section{References}

1. Richardson, S.; Hirsch, J.S.; Narasimhan, M.; Crawford, J.M.; McGinn, T.; Davidson, K.W.; Northwell COVID-19 Research Consortium. Presenting characteristics, comorbidities and outcomes among 5,700 patients hospitalized with COVID-19 in the New York City area. JAMA 2020, 323, 2052-2059. [CrossRef]

2. Duan, K.; Liu, B.; Li, C.; Zhang, H.; Yu, T.; Qu, J.; Zhou, M.; Chen, L.; Meng, S.; Hu, Y.; et al. Effectiveness of convalescent plasma therapy in severe COVID-19 patients. Proc. Natl. Acad. Sci. USA 2020, 117, 9490-9496. [CrossRef]

3. Zhou, F.; Yu, T.; Du, R.; Fan, G.; Liu, Y.; Liu, Z.; Xiang, J.; Wang, Y.; Song, B.; Gu, X.; et al. Clinical course and risk factors for mortality of adult inpatients with COVID-19 in Wuhan, China: A retrospective cohort study. Lancet 2020, 395, 1054-1062. [CrossRef]

4. Tan, B.K.; Mainbourg, S.; Friggeri, A.; Bertoletti, L.; Douplat, M.; Dargaud, Y.; Grange, C.; Lobbes, H.; Provencher, S.; Lega, J.-C. Arterial and venous thromboembolism in COVID-19: A study-level meta-analysis. Thorax 2021, 76, 970-979. [CrossRef] [PubMed]

5. Mai, V.; Tan, B.K.; Mainbourg, S.; Potus, F.; Cucherat, M.; Lega, J.-C.; Provencher, S. Venous thromboembolism in COVID-19 compared to non-COVID-19 cohorts: A systematic review with meta-analysis. Vasc. Pharmacol. 2021, 139, 106882. [CrossRef]

6. Miró, Ò.; Jiménez, S.; Mebazaa, A.; Freund, Y.; Burillo-Putze, G.; Martín, A.; Martín-Sánchez, F.J.; García-Lamberechts, E.J.; Alquézar-Arbé, A.; Jacob, J.; et al. Pulmonary embolism in patients with COVID-19: Incidence, risk factors, clinical characteristics, and outcome. Eur. Heart J. 2021, 24, 1148.

7. Liu, P.P.; Blet, A.; Smyth, D.; Li, H. The science underlying COVID-19: Implications for the cardiovascular system. Circulation 2020, 142, 68-78. [CrossRef]

8. Hendren, N.S.; Drazner, M.H.; Bozkurt, B.; Cooper, L.T., Jr. Description and proposed management of the acute COVID-19 cardiovascular syndrome. Circulation 2020, 141, 1903-1914. [CrossRef]

9. World Health Organization. COVID-19 Clinical Management: Living Guidance; 2021. 25 January 2021, COVID-19: Clinical Care. Available online: https:/ / www.who.int/publications/i/item/WHO-2019-nCoV-clinical-2021-1 (accessed on 29 September 2021).

10. National Institutes of Health. Coronavirus Disease 2019 (COVID-19) Treatment Guidelines. 2021. Available online: https: / / files.covid19treatmentguidelines.nih.gov/guidelines/covid19treatmentguidelines.pdf (accessed on 29 September 2021).

11. Wu, Z.; McGoogan, J.M. Characteristics of and Important Lessons from the Coronavirus Disease 2019 (COVID-19) Outbreak in China: Summary of a Report of 72,314 Cases from the Chinese Center for Disease Control and Prevention. JAMA 2020, 323, 1239-1242. [CrossRef]

12. Wongvibulsin, S.; Garibaldi, B.T.; Antar, A.A.; Wen, J.; Wang, M.-C.; Gupta, A.; Bollinger, R.; Xu, Y.; Wang, K.; Betz, J.F.; et al. Development of severe COVID-19 adaptive risk predictor (SCARP), a calculator to predict severe disease or death in hospitalized patients with COVID-19. Ann. Intern. Med. 2021. [CrossRef]

13. Zou, X.; Li, S.; Fang, M.; Hu, M.; Bian, Y.; Ling, J.; Yu, S.; Jing, L.; Li, D.; Huang, J. Acute Physiology and Chronic Health Evaluation II Score as a Predictor of Hospital Mortality in Patients of Coronavirus Disease 2019. Crit. Care Med. 2020, 48, 657-665. [CrossRef]

14. Iqbal, F.M.; Lam, K.; Sounderajah, V.; Clarke, J.M.; Ashrafian, H.; Darzi, A. Characteristics and predictors of acute and chronic post-COVID syndrome: A systematic review and meta-analysis. EClinicalMedicine 2021, 36, 100899. [CrossRef]

15. Ruan, Q.; Yang, K.; Wang, W.; Jiang, L.; Song, J. Clinical predictors of mortality due to COVID-19 based on an analysis of data of 150 patients from Wuhan, China. Intensive Care Med. 2020, 46, 846-848. [CrossRef] [PubMed]

16. Chinnadurai, R.; Ogedengbe, O.; Agarwal, P.; Money-Coomes, S.; Abdurrahman, A.Z.; Mohammed, S.; Kalra, P.A.; Rothwell, N.; Pradhan, S. Older age and frailty are the chief predictors of mortality in COVID-19 patients admitted to an acute medical unit in a secondary care setting- a cohort study. BMC Geriatr. 2020, 20, 409. [CrossRef] [PubMed]

17. Aziz, M.; Haghbin, H.; Lee-Smith, W.; Goyal, H.; Nawras, A.; Adler, D.G. Gastrointestinal predictors of severe COVID-19: Systematic review and meta-analysis. Ann. Gastroenterol. 2020, 33, 615-630. [CrossRef] 
18. Knight, S.R.; Ho, A.; Pius, R.; Buchan, I.; Carson, G.; Drake, T.M.; Dunning, J.; Fairfield, C.J.; Gamble, C.; Green, C.A.; et al. Stratification of patients admitted to hospital with covid-19 using the ISARIC WHO Clinical Characterization Protocol: Development and validation of the 4-C Mortality Score. BMJ 2020, 370, m3339. [CrossRef]

19. Caricchio, R.; Gallucci, M.; Dass, C.; Zhang, X.; Gallucci, S.; Fleece, D.; Bromberg, M.; Criner, G.J.; Temple University COVID-19 Research Group. Preliminary predictive criteria for COVID-19 cytokine storm. Ann. Rheum. Dis. 2021, 80, 88-95. [CrossRef]

20. Lim, S.; Shin, S.M.; Nam, G.E.; Jung, C.H.; Koo, B.K. Proper Management of People with Obesity during the COVID-19 Pandemic. J. Obes. Metab. Syndr. 2020, 29, 84-98. [CrossRef] [PubMed]

21. Di Angelantonio, E.; Bhupathiraju, S.N.; Wormser, D.; Gao, P.; Kaptoge, S.; De Gonzalez, A.B.; Cairns, B.J.; Huxley, R.; Jackson, C.L.; Joshy, G.; et al. Body-mass index and all-cause mortality: Individual-participant-data meta-analysis of 239 prospective studies in four continents. Lancet 2016, 388, 776-786. [CrossRef]

22. Sattar, N.; McInnes, I.B.; McMurray, J.J.V. Obesity Is a Risk Factor for Severe COVID-19 Infection: Multiple Potential Mechanisms. Circulation 2020, 142, 4-6. [CrossRef]

23. Report of the WHO-China Joint Mission on Coronavirus Disease 2019 (COVID-19); World Health Organization: Geneva, Switzerland, 2020 Available online: https:/ / www.who.int/docs/default-source/coronaviruse/who-china-joint-mission-on-covid-19-final-report.pdf (accessed on 29 September 2021).

24. Sun, Y.; Dong, Y.; Wang, L.; Xie, H.; Li, B.; Chang, C.; Wang, F.-S. Characteristics and prognostic factors of disease severity in patients with COVID-19: The Beijing experience. J. Autoimmun. 2020, 112, 102473. [CrossRef]

25. Gotsman, I.; Zwas, D.; Planer, D.; Admon, D.; Lotan, C.; Keren, A. The significance of serum urea and renal function in patients with heart failure. Medicine 2010, 89, 197-203. [CrossRef] [PubMed]

26. Brisco, M.A.; Zile, M.; ter Maaten, J.M.; Hanberg, J.S.; Wilson, F.; Parikh, C.; Testani, J.M. The risk of death associated with proteinuria in heart failure is restricted to patients with an elevated blood urea nitrogen to creatinine ratio. Int. J. Cardiol. 2016, 215, 521-526. [CrossRef] [PubMed]

27. Cheng, Y.; Luo, R.; Wang, K.; Zhang, M.; Wang, Z.; Dong, L.; Li, J.; Yao, Y.; Ge, S.; Xu, G. Kidney disease is associated with in-hospital death of patients with COVID-19. Kidney Int. 2020, 97, 829-838. [CrossRef] [PubMed]

28. Murata, A.; Kasai, T.; Matsue, Y.; Matsumoto, H.; Yatsu, S.; Kato, T.; Suda, S.; Hiki, M.; Takagi, A.; Daida, H. Relationship between blood urea nitrogen-to-creatinine ratio at hospital admission and long-term mortality in patients with acute decompensated heart failure. Heart Vessel. 2018, 33, 877-885. [CrossRef]

29. Chu, D.K.; Kim, L.H.; Young, P.J. Mortality and morbidity in acutely ill adults treated with liberal versus conservative oxygen therapy (IOTA): A systematic review and meta-analysis. Lancet 2018, 391, 1693-1705. [CrossRef]

30. Mellado-Artigas, R.; Ferreyro, B.L.; Angriman, F.; Hernández-Sanz, M.; Arruti, E.; Torres, A.; Villar, J.; Brochard, L.; Ferrando, C. High-flow nasal oxygen in patients with COVID-19-associated acute respiratory failure. Crit. Care 2021, 25, 58. [CrossRef]

31. Liu, Y.; Zhang, C.; Huang, F.; Yang, Y.; Wang, F.; Yuan, J.; Zhang, Z.; Qin, Y.; Li, X.; Zhao, D.; et al. 2019-novel Coronavirus (2019-nCoV) Infections Trigger an Exaggerated Cytokine Response Aggravating Lung Injur. Sci. China Life Sci. 2020, 63, 364-374. [CrossRef]

32. Chen, X.; Zhao, B.; Qu, Y.; Chen, Y.; Xiong, J.; Feng, Y. Detectable serum SARS-CoV-2 viral load (RNAaemia) is closely associated with drastically elevated interleukin 6 (IL-6) level in critically ill COVID-19 patients. Clin Infect Dis. 2020, 17, ciaa449.

33. Paranjpe, I.; Fuster, V.; Lala, A.; Russak, A.J.; Glicksberg, B.S.; Levin, M.A.; Charney, A.W.; Narula, J.; Fayad, Z.A.; Bagiella, E.; et al. Association of treatment dose anticoagulation with in-hospital survival among hospitalized patients with COVID-19. J. Am. Coll. Cardiol. 2020, 76, 122-124. [CrossRef]

34. Boyle, A.J.; Di Gangi, S.; Hamid, U.I.; Mottram, L.-J.; McNamee, L.; White, G.; Cross, L.M.; McNamee, J.J.; O’Kane, C.M.; McAuley, D.F. Aspirin therapy in patients with acute respiratory distress syndrome (ARDS) is associated with reduced intensive care unit mortality: A prospective analysis. Crit. Care 2015, 19, 109. [CrossRef] [PubMed]

35. Chen, W.; Janz, D.R.; Bastarache, J.A.; May, A.K.; O'Neal, H.R., Jr.; Bernard, G.R.; Ware, L.B. Prehospital aspirin use is associated with reduced risk of acute respiratory distress syndrome in critically ill patients: A propensity-adjusted analysis. Crit. Care Med. 2015, 43, 801-807. [CrossRef] [PubMed]

36. Erlich, J.M.; Talmor, D.S.; Cartin-Ceba, R.; Gajic, O.; Kor, D.J. Prehospitalization antiplatelet therapy is associated with a reduced incidence of acute lung injury: A population-based cohort study. Chest 2011, 139, 289-295. [CrossRef] [PubMed]

37. Kor, D.J.; Carter, R.E.; Park, P.K.; Festic, E.; Banner-Goodspeed, V.M.; Hinds, R.; Talmor, D.; Gajic, O.; Ware, L.B.; Gong, M.N.; et al. Effect of aspirin on development of ARDS in at-risk patients presenting to the emergency department: The LIPS-A randomized clinical trial. JAMA 2016, 315, 2406-2414. [CrossRef] [PubMed]

38. Kor, D.J.; Erlich, J.; Gong, M.N.; Malinchoc, M.; Carter, R.E.; Gajic, O.; Talmor, D.S. Association of prehospitalization aspirin therapy and acute lung injury: Results of a multicenter international observational study of at-risk patient. Crit. Care Med. 2011, 39, 2393-2400. [CrossRef] [PubMed] 